\title{
Swelling of two-dimensional polymer rings by trapped particles
}

\author{
E. Haleva and H. Diamant ${ }^{\mathrm{a}}$
}

School of Chemistry, Raymond \& Beverly Sackler Faculty of Exact Sciences, Tel Aviv University, Tel Aviv 69978, Israel

Original article: Eur. Phys. J. E 21, 33-40 (2006) DOI: 10.1140/epje/i2006-10041-1

Received 11 February 2008

Published online: 12 March 2008 - C EDP Sciences / Società Italiana di Fisica / Springer-Verlag 2008

We have found a technical error in Appendix A. This error affects equations (25-27) in the body of the paper and the resulting asymptotic curve in Figure 3. There is no effect on any of the qualitative results and conclusions as presented in the original paper.

The error lies in the stationary-phase approximation leading from equation (A.5) to equation (A.6). The second term in the exponent, arising from the second term in the asymptotic expansion of the modified Bessel function (Eq. (A.3)), is actually negligible compared to the $(p N)^{-N / 2}$ factor. We rewrite equation (A.5) as

$$
Z^{\mathrm{FJ}}\left(N, A \lesssim A_{\max }\right) \sim \int \mathrm{d} p e^{i p\left(A_{\max }-A\right)}(p N)^{-N / 2}
$$

which, upon a simple change of variables (or, alternatively, a stationary-phase approximation), readily yields

$$
Z^{\mathrm{FJ}}\left(N, A \lesssim A_{\max }\right) \sim\left(A_{\max }-A\right)^{N / 2}
$$

Thus, the $N / 4$ exponent appearing in equations (A.6) and (A.7) of the original paper should be replaced with $N / 2$. The same holds for equation (25) of Section 4.1.3,

$$
P_{0}^{\mathrm{FJ}}\left(N, A \lesssim A_{\max }\right) \sim\left(A_{\max }-A\right)^{N / 2}
$$

This leads to the following corrected versions of equations (26) and (27):

$$
\begin{aligned}
& Z^{\mathrm{FJ}}(N, Q \gg N)=\left(A_{\max }\right)^{Q+N / 2} \frac{\Gamma(1+N / 2)}{\Gamma(1+N / 2+Q)} \\
& \left\langle A^{\mathrm{FJ}}(N, \hat{Q} \gg 1)\right\rangle \simeq A_{\max } \frac{\hat{Q}}{\hat{Q}+1 / 2} \simeq A_{\max }\left(1-\frac{1}{2 \hat{Q}}\right) .
\end{aligned}
$$

Thus, the exact approach of the mean area to its maximum value differs from the mean-field result, equation (22), by a factor of $1 / 2($ not $1 / 4)$. Finally, Figure 3 is redrawn on the following page with the correct asymptote (corrected Eq. (27)).

\footnotetext{
a e-mail: hdiamant@tau.ac.il
} 


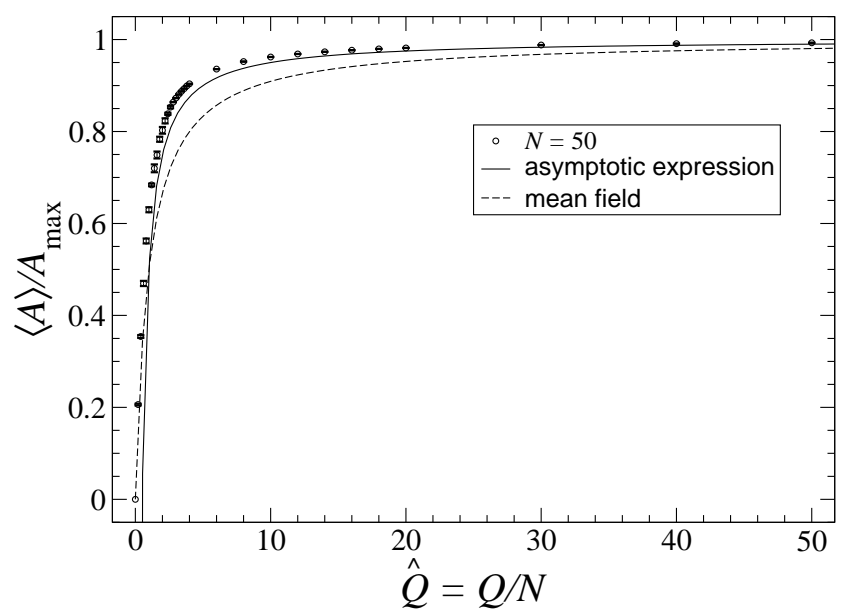

Fig. 3. Mean area of a freely jointed ring, scaled by $A_{\max }$, as a function of the rescaled particle number, as obtained by Monte Carlo simulations for $N=50$ (symbols with error bars). The solid line shows the exact asymptote for a highly swollen ring (Eq. (27)). The dashed line presents the mean-field result (Eq. (22)). 